\title{
Eye-hand coordination during online reach corrections is task-dependent
}

\author{
Anouk J. de Brouwer ${ }^{1} \&$ Miriam Spering ${ }^{1,2,3}$ \\ ${ }^{1}$ Department of Ophthalmology \& Visual Sciences, University of British Columbia, Vancouver, \\ BC, Canada \\ ${ }^{2}$ Institute for Computing, Information and Cognitive Systems, University of British Columbia, \\ Vancouver, BC, Canada \\ ${ }^{3}$ Djavad Mowafaghian Centre for Brain Health, University of British Columbia, Vancouver, BC, \\ Canada
}

Running title: Eye-hand coordination during online reach corrections

Corresponding author: Miriam Spering, miriam.spering@ubc.ca

\section{Abstract}

To maintain accurate movements, the motor system needs to deal with errors that can occur due to inherent noise, changes in the body, or disturbances in the environment. Here, we investigated the temporal coordination of rapid corrections of the eye and hand in response to a change in visual target location during the movement. In addition to a 'classic' double-step task in which the target stepped to a new position, participants performed a set of modified double-step tasks in which the change in movement goal was indicated by the appearance of an additional target, or by a spatial or symbolic cue. We found that both the absolute correction latencies of the eye and hand and the relative eye-hand correction latencies were dependent on the visual characteristics of the target change, with increasingly longer latencies in tasks that required more visual and cognitive processing. Typically, the hand started correcting slightly earlier than the eye, especially when the target change was indicated by a symbolic cue, and in conditions where visual feedback of the hand position was provided during the reach. Our results indicate that the oculomotor and limb-motor system can be differentially influenced by processing requirements of the task and emphasize that temporal eye-hand coordination is flexible rather than rigid.

Key words: online control; visuomotor; perturbation; reaction time; reaching; eye movements 


\section{Introduction}

2 How humans adjust and optimize movements to correct for errors that are due to sensory and motor noise, changes in the body, or external disturbances is a major focus of current neuroscience research. In the laboratory, tasks that artificially produce movement errors have revealed the ability to rapidly correct for errors during the movement (i.e., movement corrections), as well as the ability to adjust the movement to consistent errors over the course of several repetitions (i.e., motor adaptation). Here, we investigate the temporal coordination of movement corrections in eye and hand movements in a reaching task.

The double-step task has been widely used to study both movement corrections and motor adaptation in eye and hand movements. In this classic paradigm, the visual target is displaced at the time of movement onset to simulate a spatial error. In the case of saccadic eye movements, the endpoint error after the saccade toward the initial target location triggers a second, corrective saccade to the new target location (Becker and Jürgens 1979; Hallett and Lightstone 1976; Joiner et al. 2010; Tian et al. 2013). If the target is repeatedly displaced to the same location, motor adaptation will result in an adjustment of the initial saccade (Herman et al. 2013; McLaughlin 1967; Tian et al. 2009). The double-step paradigm has also been used extensively to study corrections of reach movements, as well as to study adaptation of reach movements (Magescas and Prablanc 2006). Because the duration of reach movements is much longer than that of saccades, movement corrections in response to a displacement of the reach target typically occur online (i.e., 'in flight') (Georgopoulos et al. 1981; Goodale et al. 1986; Megaw 1974; Soechting and Lacquaniti 1983) with reaction times as short as $110 \mathrm{~ms}$ (Brenner and Smeets 1997; Day and Lyon 2000; Prablanc and Martin 1992). Another popular paradigm to study reach corrections is the cursor displacement paradigm, in which hand movements correct within short latencies to a perceived deviation of the reach trajectory (Brenner and Smeets 2003; Franklin and Wolpert 2008; Sarlegna et al. 2003; Saunders and Knill 2003). Together, these findings indicate that visual information is continuously used to control and correct movements.

The significant progress in understanding how the sensorimotor system corrects for errors in eye and hand movements is largely based on studies that have investigated these two motor systems separately. The few studies that measured both eye and hand movements in response to a displacement of a visual target have made several interesting observations. First, hand movement corrections start earlier when a corrective saccade accompanies the reach, as opposed to when the eyes are instructed to fixate (Abekawa et al. 2014; Diedrichsen et al. 2004), 
showing a facilitative effect of eye movements on hand movements. Second, whereas the eye usually leads the hand by (several) hundred milliseconds when initiating a goal-directed reach (e.g., Land and Hayhoe 2001; Prablanc et al. 1979), the hand might start correcting for spatial errors before the eye (Abekawa et al. 2014; Gritsenko et al. 2009), although the opposite finding (i.e., the eye corrects before the hand) has also been reported (Neggers and Bekkering 2002). Here, we investigated the detailed temporal coordination of eye and hand movement corrections by assessing the timing of corrections in various stimulus and task conditions. For hand movements, it has been shown that the visual characteristics of the target (Kozak et al. 2019; Veerman et al. 2008), as well as the presence of visual distractors (Reichenbach et al. 2014) can influence the duration within which corrections are initiated, but eye movements were either not measured (Veerman et al. 2008) or participants were instructed to fixate (Reichenbach et al. 2014) in these studies.

On one hand, we might expect a tight temporal coupling of corrective eye and hand movements, independent of stimulus and task conditions, based on the close behavioral and neurophysiological connection of both movements (de Brouwer et al. 2021; Land and Hayhoe 2001; Prablanc et al. 1979). On the other hand, the temporal coupling might differ across conditions. For example, recent work has shown that errors in the reach trajectory (i.e., due to a cursor jump) evoke the earliest and most vigorous corrections when gaze is directed at the reach target, as compared to surrounding locations (de Brouwer et al. 2018). As such, looking at the target as early as possible might be particularly important when peripheral visual feedback of the hand can be used to improve reach accuracy (i.e., when the hand is visible). In other conditions, it might be optimal to maintain fixation at the original target to prevent a temporary disturbance of the retinal image by eye movements (Mrotek and Soechting 2007; Neggers and Bekkering 2000; Ross et al. 2001), and perform a reach correction in peripheral vision.

Participants performed a set of tasks in which they were asked to reach, as rapidly and accurately as possible, toward a visual target using a robotic manipulandum, while their hand and eye movements were recorded. In a subset of trials, an unpredictable change in the movement goal occurred. We included a classic double-step task (Hallett and Lightstone 1976), in which the target 'stepped' to a new location, triggering an immediate, reactive saccade to that location. In addition, we designed a set of modified double-step tasks with the intention to manipulate the latency of the saccade to the new movement goal. In these tasks, the change in movement goal was indicated by the appearance of an additional target, or by a spatial or symbolic cue, placing

65 different demands on visual and cognitive processing. Whereas the visual target displacement could be expected to trigger an immediate, reactive saccade, the additional target and cue 
conditions were designed to prevent triggering an immediate saccade and instead produce a later, voluntary saccade. Thus, we hypothesized that our modified double-step tasks would delay the corrective saccade to the new goal location, but the question remained whether this would also delay the reach correction (Abekawa et al. 2014; Diedrichsen et al. 2004), or whether the reach correction would be prioritized to be completed as rapidly as possible. In addition to varying the visual characteristics of the target change, we manipulated the presence of visual feedback of the hand. We hypothesized that the presence of hand feedback would speed up the corrective saccade to allow optimal monitoring of the reach trajectory (de Brouwer et al. 2018), and potentially speed up the reach correction (Reichenbach et al. 2009). Overall, we aim to contribute to a better understanding of the temporal coordination of eye and hand movements during online reach corrections, the role of visual and cognitive demands in different task contexts, and the role of visual feedback.

\section{Methods}

81 Nineteen participants (mean age 26 years, range 19-36 years, 5 female) completed the 82 experiment and were compensated \$12/hour for their participation. Three other participants did 83 not complete the experiment because the experimenter could not achieve sufficiently accurate 84 calibration of the eye tracker (mean error $<1.5^{\circ}$ and maximum error $<3.0^{\circ}$ visual angle). All 85 participants were self-reported right-handed and had normal or corrected-to-normal visual acuity. 86 The study was approved by the University of British Columbia Behavioural Research Ethics

87 Board. Participants provided written informed consent before the start of the experiment. the analysis because they had less than 4 (out of 10) valid trials for any combination of task $X$ target change time $\times$ target change direction in two or more tasks. The most common reason for

91 an insufficient number of valid trials was that an initial saccade to the target could not be detected,

92 likely due to eye tracking difficulties. This exclusion resulted in 16 complete and analyzed data 93 sets for the experiment. 
94 Experimental setup

95 Participants performed reaching movements to visual targets with their right hand. They were 96 seated in a chair with their forehead resting against a pad and their hand holding on to the handle 97 of a robotic manipulandum that moves in the horizontal plane (KINARM End-Point Robot; BKIN 98 Technologies Ltd., Kingston, ON, Canada). A mirror was mounted horizontally above the handle, and an LCD monitor was mounted horizontally above the mirror, such that stimuli were projected in the plane of the handle when looking onto the mirror while the view of the arm was blocked (Fig. 1A). Kinematics of the handle were recorded with a temporal resolution of $1129 \mathrm{~Hz}$, resampled to $1000 \mathrm{~Hz}$. Eye movements of the left eye were recorded using a built-in monocular video-based eye tracker (Eyelink 1000, SR Research Ltd., Kanata, ON, Canada) with a temporal resolution of $500 \mathrm{~Hz}$, resampled to $1000 \mathrm{~Hz}$. The pupil was detected with a proprietary algorithm that accounted for small head movements, which were measured using a target sticker placed on the participant's forehead or cheek. The eye tracker was calibrated for the 2D horizontal workspace using proprietary algorithms (BKIN Technologies).

\section{Task and visual stimuli}

109 Figure 1B provides a schematic illustration of the tasks. The hand position was represented on 110 the screen as a cursor ( $1 \mathrm{~cm}$ diameter white circle) aligned with the handle. All other stimuli were 111 presented in red $\left(5.9 \mathrm{~cd} / \mathrm{m}^{2}\right)$ on a black background $\left(0.9 \mathrm{~cd} / \mathrm{m}^{2}\right)$ for clear, high-contrast visibility.

112 Each trial began with the presentation of a start position (2 cm diameter circle) at the horizontal 113 center of the screen near the participant. Participants were instructed to move the cursor and their 114 gaze to the start position to initiate the trial. The cursor had to be at the start position for $250 \mathrm{~ms}$, 115 and the recorded eye position on the screen had to be within a $5 \mathrm{~cm}$ radius of the start position at 116 the end of the 250-ms period. After a random delay of 250 to $500 \mathrm{~ms}$, the start position 117 disappeared and the reach target (2 cm diameter circle) appeared $20 \mathrm{~cm}$ in front of the start 118 position and in line with the start position or $5 \mathrm{~cm}$ to the left or right of the start position. Participants 119 were instructed to reach toward the target as quickly and accurately as possible. In $40 \%$ of trials, 120 the movement goal remained unchanged during the trial. In the remaining $60 \%$ of trials, a sudden 121 change in movement goal from the central to either the left or right target location was presented. 122 Participants were instructed to move their hand to the new target as soon as possible. The target 123 disappeared $250 \mathrm{~ms}$ after detection of the reach offset, defined by the velocity of the handle falling 124 below a threshold of $2 \mathrm{~cm} / \mathrm{s}$ for $250 \mathrm{~ms}$. To encourage participants to perform fast reaching 
125 movements throughout the experiment, a message 'Too slow' was displayed on the screen if the

126 movement time was longer than $800 \mathrm{~ms}$ in unperturbed trials, or longer than $1100 \mathrm{~ms}$ in trials with

127 a change in movement goal. A new trial started after a $500 \mathrm{~ms}$ inter-trial-interval.

128 Participants performed five versions of the main task, in separate blocks (Fig. 1B). (1) In

129 the classic double-step task, a single reach target stepped from the central to the left or right

130 target position. (2) In the placeholder task, placeholders (2 cm diameter open circles) were 131 presented in addition to the target at the two non-target locations. When the target stepped, it 132 would 'swap' position with one of the placeholders. (3) The overlap task was similar to the 133 placeholder task, except that when the target stepped, the central circle would remain filled (i.e., 134 the old target would stay in its position when the new target was presented). $(4,5)$ In the line and 135 arrow cue tasks, three filled targets were presented at the three target locations. (4) In the line 136 (i.e., spatial) cue task, a reach to the left or right target, or a change in movement goal from the 137 central to the left or right target, was indicated by the appearance of a vertical line (10 $\mathrm{cm} \times 0.2$ $\mathrm{cm}$ ) presented $10 \mathrm{~cm}$ to the left or right of the midline, and vertically centered between the start and target position. (5) In the arrow (i.e., symbolic) cue task, a reach or change to the left or right target was indicated by the central circle changing into a leftward or rightward pointing triangle $(2.5 \times 1.5 \mathrm{~cm})$.

To ensure that the time of the change was unpredictable and that our results were not determined by the time of the target change, the target jump or appearance of the cue was

144 triggered by one of three events: the onset of the saccade ( $y$ gaze position reached a third of the 145 distance between the start and target position), the onset of the reach movement (the cursor had 146 fully moved out of the start position), or the time where the handle passed the midpoint between 147 the start position and the target position. The execution of the saccade and the onset of the reach 148 movement are commonly used target change triggers in target displacement tasks (e.g., 149 Diedrichsen et al. 2004; Gritsenko et al. 2009; Prablanc and Martin 1992; Oostwoud Wijdenes et 150 al. 2013). We included the late target change time to make sure that the timing of the corrective 151 saccade would not be influenced by the saccade refractory period, which is commonly observed 152 to be around $150 \mathrm{~ms}$ (Carpenter 1977). 
A

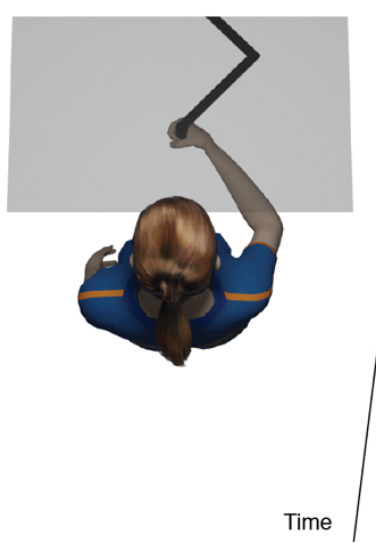

B
1) Double-step task

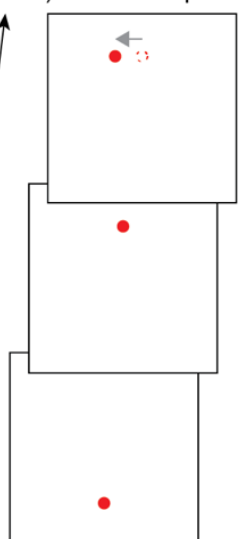

2) Placeholder task

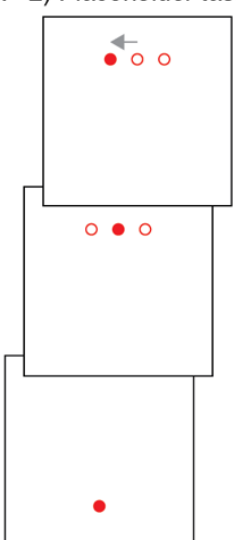

3) Overlap task

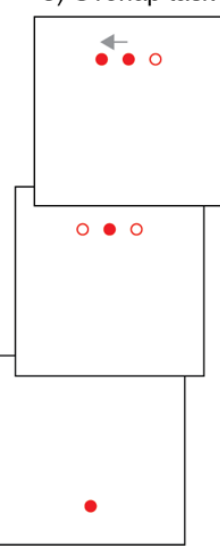

4) Line cue task

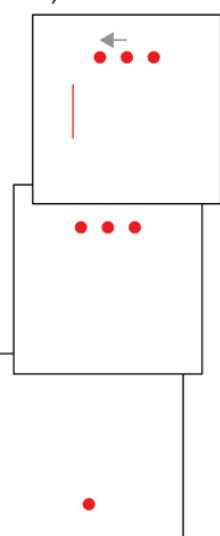

5) Arrow cue task

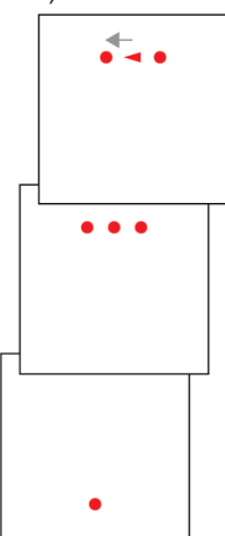

Figure 1. Experimental setup and tasks. A) Participants performed reaching movements in the horizontal plane using a KINARM robotic manipulandum. Vision of the hand was blocked by a mirror onto which the stimuli were projected such that they appeared in the plane of the handle. The mirror is depicted as transparent to illustrate the location of the hand and the manipulandum. B) In each task, participants moved their hand from a start position to one of three target positions $20 \mathrm{~cm}$ in front of the start position (both shown as red circles). In a subset of trials, the movement goal changed from the central position to a position $5 \mathrm{~cm}$ to the left or right of the central position (shown by the grey arrow pointing to the left that was not visible to the participant). In the doublestep task (1), the target stepped from the central to the left/right position (the dotted circle indicates the original, invisible target position). The placeholder task (2) was identical to the double-step task, except that the two alternative target positions were indicated with open circles together with the target position. The overlap task (3) was identical to the placeholder task, except that the original target position remained 'filled in' when the target jumped to its new position. In the line cue task (4), all three possible target locations were indicated, and the (new) target was indicated by a spatial cue: a line on the left for the left target, a line on the right for the right target, no line for the central target). In the arrow cue task (5), the (new) target was indicated by a symbolic cue location at the central target: a leftward pointing arrow for the left target, a rightward pointing arrow for the right target, a circle for the central target.

Each participant performed one practice block and ten experimental blocks, with short breaks in between blocks. Half of the experimental blocks were performed with online visual feedback of the hand position during the reach, and the other half was performed with endpoint feedback only. In the latter case, the cursor disappeared when the reach target was presented and reappeared after movement offset. The order of tasks and feedback was counterbalanced. Twenty different orders for the five tasks were created by starting with a $5 \times 5$ Latin Square in which every task occurs once in each row and once in each column, and then duplicating this square three times while randomly permuting the columns. Half of the participants performed every task first with online cursor feedback and then with endpoint cursor feedback, while the other half performed 
every task first with endpoint cursor feedback and then with online cursor feedback. The eye tracker was typically recalibrated at the start of each block, however, in some cases where calibration proved difficult, the eye tracker was recalibrated every two blocks. No instructions on eye movements were given except for directing the eyes to the start position at the beginning of the trial. The practice block consisted of 20 trials without a target change. Each experimental block consisted of 20 repetitions of unperturbed trials to the central target, 10 repetitions of unperturbed trials to the left/right target, 10 repetitions of trials in which the movement goal changed to the left/right during the saccade to the target, 10 repetitions of trials in which the movement goal changed to the left/right target following reach onset, and 10 repetitions of trials in which the movement goal changed to the left/right following the handle passing the point midway between the start and target position, resulting in a total of 100 trials per block. Participants took about 90 minutes to complete the experiment.

The timing of the appearance of all visual stimuli and events (start position, target, target displacement, cue) was corrected offline by the delay in the system ( $57 \pm 5 \mathrm{~ms})$, which was measured using a photodiode after the completion of data collection. Trials were excluded if the offline analysis showed that the target change had occurred before the event that triggered it $(<0.5 \%$ of trials).

\section{Hand and eye movement data preprocessing}

201 The $x$ and $y$ positions of the center of the handle in the horizontal plane were used for the analysis of hand movements. For each trial, the onset of the reach was defined as the first moment in time where the resultant velocity of the handle was greater than $5 \mathrm{~cm} / \mathrm{s}$. In the offline analysis, the reach offset was defined as the first moment in time where the resultant velocity of the handle was smaller than $5 \mathrm{~cm} / \mathrm{s}$ for $250 \mathrm{~ms}$ after the handle had passed the point midway between the start and target position. Trials were discarded if the onset of the reach occurred before the target appeared ( $1 \%$ of trials), or more than $1000 \mathrm{~ms}$ after the target appeared $(<0.5 \%)$. Trials were also

208 discarded if the $y$ amplitude of the reach was less than half the target distance, or the $x$ amplitude 209 was less than half of the target displacement in change trials (1\%).

The $x$ and $y$ eye positions on the screen, as obtained from the calibrated eye tracker, were

211 used for analysis of the eye movements. Eye blinks, intervals in which the pupil signal was

212 missing, and intervals in which the eye was detected outside the screen were removed from the 
$213 x$ and $y$ eye positions. For intervals with missing data for less than $100 \mathrm{~ms}$ (i.e., shorter than a 214 typical blink duration), $x$ and $y$ eye position were linearly interpolated. Eye positions were filtered 215 with a 2nd order lowpass Butterworth filter with a cut-off frequency of $30 \mathrm{~Hz}$. $X$ and $y$ eye velocity $216(\dot{x}, \dot{y})$ were computed by numerically differentiating $x$ and $y$ eye position signals.

Next, 2D eye positions on the screen were converted to an eye-based 3D coordinate system (Singh, Perry \& Herter, 2016), assuming that the height of the stimulus plane relative to the eye is fixed (i.e., $z$ is constant). The 3D eye positions on the screen were then transformed to an eye-based spherical coordinate system according to

$$
\begin{gathered}
\rho=\sqrt{x^{2}+y^{2}+z^{2}} \\
\theta=\tan ^{-1}\left(\frac{y}{x}\right) \\
\phi=\cos ^{-1}\left(\frac{z}{\rho}\right)
\end{gathered}
$$

with $\rho$ the radial distance from the eye to the point of gaze on the screen, $\theta$ the azimuthal angle in the $x y$-plane, and $\phi$ the elevation angle. Next, $\rho, \theta$, and $\phi$ were differentiated to obtain $\dot{\rho}, \dot{\theta}$ and $\dot{\phi}:$

$$
\dot{\rho}=\frac{x \cdot \dot{x}+y \cdot \dot{y}+z \cdot \dot{z}}{\rho}
$$

Note that $\dot{z}$ is assumed to be zero, reducing some parts of the equations to zero. Finally, the eye angular velocity $\omega$ was calculated according to

$$
\omega=\sqrt{(\dot{\theta} \cdot \sin (\phi))^{2}+\dot{\phi}^{2}}
$$

Saccades were detected by searching for intervals in which angular eye velocity was larger than $100 \%$ for at least $4 \mathrm{~ms}$. Next, saccade onset was defined as the last sample in which eye velocity was below a threshold of $30 \% \mathrm{~s}$ for $2 \mathrm{~ms}$. Saccade offset was defined as the first sample in which eye velocity fell below a threshold of $30 \%$ for $2 \mathrm{~ms}$.

Target saccades were defined as saccades that started between 50 ms before (predictive saccades) and $1000 \mathrm{~ms}$ after target appearance, with a $y$ onset position before the midpoint between start and target position, and a minimum $y$ amplitude of half the target distance. Trials were discarded if a target saccade was not detected ( $4 \%$ of trials). Corrective saccades from the initial to the final target were defined as saccades that started at least $50 \mathrm{~ms}$ after the target 
242 change, with a $y$ onset and offset position past the midpoint between start and target position,

243 and a minimum $x$ amplitude of half the target displacement. Change trials were discarded if a

244 corrective saccade was not detected (1\% of trials). $93 \%$ of trials were included in the analysis.

\section{Hand and eye movement analysis}

246 We computed the latencies of the initial saccade and reach movements to the target and, for 247 target change trials, the latencies of the corrective responses of the eye and hand. The latency of 248 the corrective saccade was defined as the latency of the first saccade directed towards the new 249 target position following the target change. The latency of the corrective hand movement was 250 defined using a variant of the extrapolation method applied to the velocity of the hand in the $x$ 251 direction (i.e., the direction of the perturbation) (Oostwoud Wijdenes et al. 2014). We first 252 corrected the $x$ velocity in individual change trials by subtracting the average $x$ velocity in no253 change trials to the central target in the same block. Next, for each trial we obtained the two points 254 at which the additional $x$ velocity reached $30 \%$ and $70 \%$ of the first peak in velocity, and fitted a 255 line through these points and all data points in between. The onset of the reach correction was 256 defined as the time where this line crossed zero. In some trials, the increase in $x$ velocity was not 257 approximately linear in the interval between the $30 \%$ and $70 \%$ peak velocity points, or there was 258 no clear, single peak in $x$ velocity, resulting in a poor fit. We therefore discarded trials in which the $259 R^{2}$ of the fit was below 0.95 or in which the extrapolated onset occurred before the target change 260 (2\% of trials).

\section{Hypotheses and statistical analysis}

262 We designed a set of double-step tasks to determine the effect of target and cue characteristics 263 on the temporal coordination of corrections of the eye and hand. We hypothesized that, with 264 respect to the classic double-step task, corrective saccade latency would increase in the 265 placeholder task, because of the necessity to identify which of the items is the displaced target 266 (Oostwoud Wijdenes et al. 2014; Smeets et al. 2016), as well as in the overlap task, as latency 267 increases are a well-known effect of stimulus overlap (Saslow 1967). We predicted a greater 268 increase in corrective saccade latency in the line and arrow cue tasks, potentially even until after 269 the cursor hit the target, due to the lack of visual stimuli triggering a reactive saccade to the new 270 target. We also hypothesized that corrective saccade latencies would be shorter when online 271 cursor feedback was provided compared to when endpoint feedback was provided, to allow the 272 earliest possible use of visual feedback to help guide the incoming cursor (de Brouwer et al., 
273 2018). If the reach and saccadic system show strong temporal coupling, we further expect a 274 constant delay between reach and saccade correction latencies across tasks, with high 275 correlations within individuals. Weak coupling would result in low correlations within individuals, 276 and potentially in varying delays between corrective responses across tasks.

For each of the latencies, we computed the median value per subject, task and feedback condition for no change trials to the central target (20 repetitions), and per subject, task, feedback condition, target change time, and target change direction for change trials (10 repetitions per trial type). These median values were averaged across left and right target change directions, resulting in four values for each combination of task and feedback. To rule out any delays in the corrective saccade due to a saccade refractory period when the target changes during or immediately after the saccade, we assessed the effect of change time on the latency of corrections in the classic double-step task. On average, the target change was visible on the screen $86 \mathrm{~ms}$ after saccade onset, $54 \mathrm{~ms}$ after reach onset, and $57 \mathrm{~ms}$ after the hand passed the midway point, or 242, 343, and 506 ms after target appearance, respectively. Although there was a significant effect of change time on corrective saccade latency, with the shortest latencies when the change occurred after reach onset, there was no significant difference between latencies when the change occurred after saccade onset and when the change occurred midway during the reach (i.e., with a long delay after the initial saccade). This suggests that factors other than the saccade refractory period determined the corrective saccade latency. We therefore averaged the data across change times, calculating the mean and within-subject standard error of the mean (Cousineau 2005) for each combination of task and feedback.

Statistical analyses were performed in $\mathrm{R}$ using the rstatix package ( $\mathrm{v}$ 0.7.0). We performed 5 (task) $\times 2$ (feedback) repeated measures ANOVAs, applying a Greenhouse-Geisser correction when the assumption of sphericity was violated, and we calculated generalized eta squared effect sizes $\left(\eta_{G}^{2}\right)$. Significant main effects were followed up by pairwise comparisons with Bonferroni correction for multiple comparisons.

\section{Results}

300 The main goal of this study was to determine the influence of the characteristics of the visual 301 target or cue that indicates a sudden change in movement goal, on the timing of rapid corrective 302 responses of the eye and hand. An additional goal was to determine the effect of feedback of the 303 hand on the timing of these corrections. In the following sections, we will first show task and 
304 feedback effects on correction latencies of the eye and hand. We then report how the temporal relation between eye and hand corrections was affected by task and feedback.

\section{Initial movement latencies}

307 Regardless of task and feedback condition, each double-step trial started with a saccade toward 308 the initial (central) target that was followed by an initial reach toward the central target. To provide 309 insight into the timeline of events in our task, we report the mean latencies of these initial 310 movements, calculated per task and feedback condition, in Table S1. Briefly, the mean latencies 311 ranged between 143 and 162 ms for initial saccades, and between 197 and 260 ms for initial 312 reach movements. Since all correction latencies in our main analyses were assessed relative to 313 the target change, which was triggered by the execution of the saccade or reach, we did not test 314 the effects of task or feedback on these initial latencies. The short initial latencies suggest that 315 participants were anticipating the appearance of the target. The Pearson correlation coefficients 316 between saccade and reach latencies, calculated separately for each participant and then 317 averaged across participants, ranged between 0.39 and 0.59 (Table S1), indicating moderate 318 temporal coupling during the initial movement phase.

\section{Effects of task and feedback on movement corrections}

321 In response to the change in movement goal, both the eye and the hand rapidly initiated a 322 correction in all conditions. Examples of these corrections and their timing are shown in Figure 2 323 for a representative participant, in blocks with online cursor feedback. Figure 2A shows that the 324 initial straight-ahead reach trajectory was followed by a correction to the left or right, with later 325 target changes (brighter colours) eliciting later trajectory adjustments requiring a greater change in reach direction. The eye and hand started correcting at around the same time (Fig. 2B), and the corrective saccade was of much shorter duration than the reach correction.

Averaging across participants revealed a clear task-dependency of correction latencies (Fig. 3A, B). Figure 3A shows that corrective saccade latencies (relative to the time of target change) were modulated by the characteristics of the visual target or cue that indicated the target change. Latencies were shortest in the classic double-step task ( $M=191 \mathrm{~ms})$, longer in the placeholder task ( $M=244 \mathrm{~ms})$, longer again in the overlap and line cue tasks $(M=271$ and 271 
334 main effect of task in a 5 (task) $\times 2$ (feedback) ANOVA $\left(F(4,60)=67.5, p<0.001, \eta_{G}^{2}=0.63\right)$. Post-

335 hoc pairwise comparisons confirmed significant differences between all tasks $(p<0.01)$, except

336 between the overlap task and the line cue task $(p=1.000$; all $p$-values for pairwise comparisons

337 here and below were adjusted with a Bonferroni correction). Corrective saccade latencies did not

338 differ between blocks with online cursor feedback and blocks with endpoint cursor feedback (main effect $\left.F(1,15)=0.2, p=0.647, \eta_{G}^{2}=0.001\right)$. There was no significant interaction between task and feedback $\left(F(4,60)=1.5, p=0.220, \eta_{G}^{2}=0.007\right)$.

341 Reach correction latencies were also dependent on the characteristics of the visual target

342 or cue that indicated the target change (Fig. 3B). Reach correction latencies were shortest in the 343 double-step task ( $M=194 \mathrm{~ms})$, longer in the placeholder task ( $M=238 \mathrm{~ms})$, and longest in the 344 overlap $(M=265 \mathrm{~ms})$, line cue $(M=266 \mathrm{~ms})$ and arrow cue $(M=280 \mathrm{~ms})$ tasks. This was reflected 345 in a significant main effect of task $\left(F(4,60)=57.7, p<0.001, \eta_{G}^{2}=0.52\right)$, with significant pairwise 346 differences between all tasks (all $p<0.001$ ), except between the overlap, line cue, and arrow cue 347 tasks $(p>0.05)$. In addition, reach correction latencies were shorter with online cursor feedback $348(M=240 \mathrm{~ms})$ than with endpoint cursor feedback $(M=258 \mathrm{~ms}$; main effect of feedback $F(1,15)=9.2$, $\left.349 p=0.008, \eta_{G}^{2}=0.08\right)$. The effect of feedback was significant for all tasks $(p<0.05)$ except the arrow 350 cue task $(p=0.375)$, as revealed by pairwise comparisons following up on the significant interaction between task and feedback $\left(F(4,60)=3.2, p=0.018, \eta_{G}^{2}=0.01\right)$.

In summary, corrective saccade latencies were longer in the modified double-step tasks 353 than in the classic double-step task, in line with our expectations. Reach correction latencies 354 followed a similar pattern to saccade latencies, with the exception that there was no further 355 increase in latency for the arrow cue task. We also found that while corrective saccade latencies 356 were not influenced by the presence of online cursor feedback, reach corrections started earlier 357 when online cursor feedback was provided. Knowing that both corrective saccade latencies and 358 reach correction latencies were affected by the task, the next question is how the relative timing 359 and the correlation between saccade and reach corrections were influenced by the task. 
A Double-step task

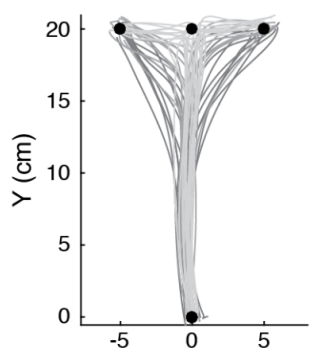

Placeholder task

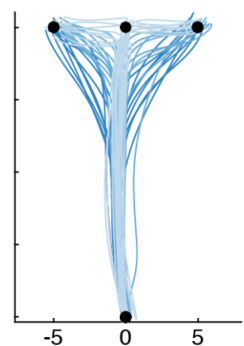

B

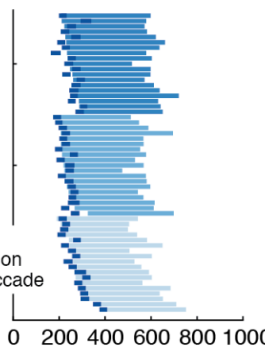

Overlap task

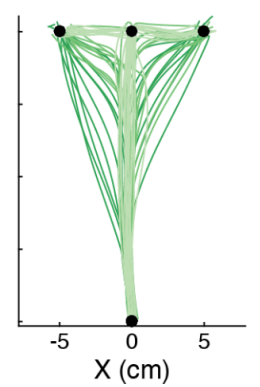

Line cue task
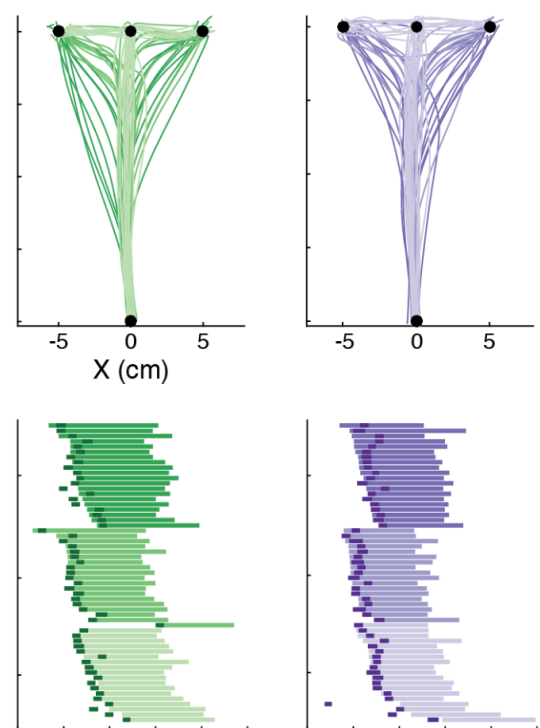

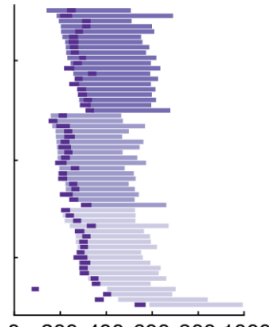

2004006008001000
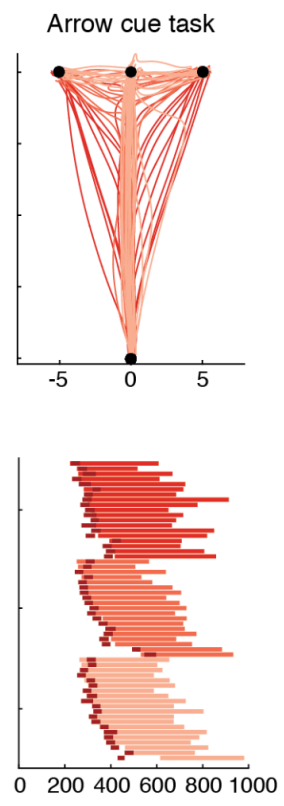
Time since target change (ms)

Figure 2. Eye and hand corrections in response to a change in movement goal in individual trials of an example participant, in tasks with online cursor feedback. A) Hand trajectories. Each trajectory is a single trial, with the brightness indicating the target change time as indicated in the legend in B. Note that the results were averaged across target change times (see Methods). B) Timing of corrections relative to the target change. Reach corrections are shown by the longer horizontal bars (one for each trial), with the brightness indicating the target change time. Corrective saccades are shown by the short dark-colored bars. For each target change time, trials are sorted according to the latency of the reach correction. 

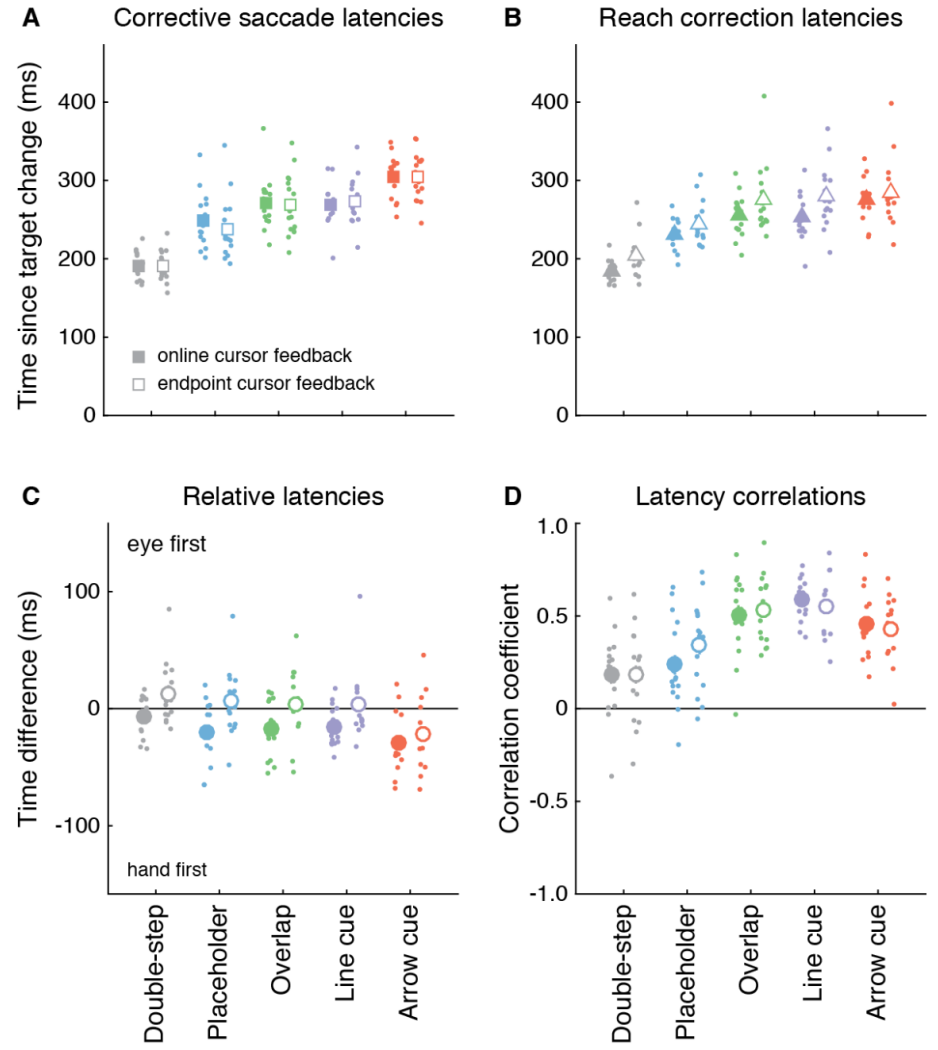

390
Figure 3. Timing of eye and hand corrections in response to a change in movement goal, as a function of task and cursor feedback. A) Latencies of corrective saccades relative to the target change. Each small dot represents a participant and each square represents the average across participants $(n=16)$. B) As A, but showing reach correction latencies. $C$ ) Differences between corrective saccade latencies and reach correction latencies, with positive values indicating that the eye started correcting first, and negative values indicating that the hand started correcting first. D) Correlations between corrective saccade and reach correction latencies. For all panels, colors represent different tasks, filled symbols represent online cursor feedback and open symbols represent endpoint feedback only. Error bars represent \pm one within-subjects standard error of the mean [note that these are often hidden behind the symbol showing the average value].

\section{Temporal coordination of movement corrections}

394 To determine the influence of task on the temporal coordination of corrections of the eye and hand, we calculated the relative latencies of corrective saccades and reach trajectory corrections, as well as the correlations between latencies. Figure $3 \mathrm{C}$ shows that the hand often started correcting before the eye. The relative latencies of corrections of the hand and eye showed a significant effect of task $\left(F(4,60)=9.8, p<0.001, \eta_{G}^{2}=0.13\right)$ and feedback $(F(1,15)=15.3, p=0.001$, $\left.\eta_{G}^{2}=0.13\right)$, as well as a significant interaction $\left(F(4,60)=3.4, p=0.014, \eta_{G}^{2}=0.01\right)$. First, the lead of the hand was greater in the arrow cue task $(M=25 \mathrm{~ms})$ than in the other four tasks $($ all $p<0.001)$. The relative latencies in the double-step, placeholder, overlap, and line cue tasks $(M=-3, M=7$, $M=6$ and $M=6 \mathrm{~ms}$, respectively) did not differ significantly between each other (all $p>0.05$ ). Second, the lead of the hand was greater when the hand cursor was visible during the reach

$404(M=18 \mathrm{~ms})$ than when the cursor was only shown at the end of the reach $(M=-1 \mathrm{~ms})$. Pairwise 405 comparisons to unpack the interaction showed that this difference was significant for all tasks (all $p<0.01)$ except the arrow cue task $(p=0.080)$. 
As an additional measure of the temporal coordination of corrections, we calculated Pearson correlation coefficients between corrective saccade and reach correction latencies (Fig. 3D). These correlations showed a significant effect of task $\left(F(4,60)=18.0, p<0.001, \eta_{G}^{2}=0.36\right)$ but not feedback $\left(F(1,15)=0.3, p=0.623, \eta_{G}^{2}=0.002\right)$, and no significant interaction $(F(4,60)=1.1$, $\left.p=0.343, \eta_{G}^{2}=0.02\right)$. Correlations were lower in the double-step task $(M=0.18)$ than in the overlap

$412(M=0.52, p<0.001)$, line cue $(M=0.57, p<0.001)$, and arrow cue tasks $(M=0.44, p<0.001)$.

413 Correlations were also lower in the placeholder task $(M=0.29)$ than in the overlap $(p=0.003)$, line 414 cue $(p<0.001)$, and arrow cue tasks $(p=0.026)$, and correlations were higher in the line cue task 415 than in the arrow cue task $(p=0.020)$.

416 In summary, the hand generally started correcting its trajectory in response to a change 417 in movement goal before a corrective saccade occurred. Although in some cases the relative 418 delays between corrections of the hand and eye were around zero, the longer neuromuscular 419 delays and higher inertia of the arm (Gribble et al. 2002) imply that even in these cases, the neural 420 signal to execute a correction occurred earlier for the hand than for the eye. This is in contrast to 421 the temporal coordination of the initiation of goal-directed eye-hand movements, where the eye 422 typically leads the hand. Taken together with the results on absolute latencies above, the greater 423 hand-eye delay in the arrow cue task compared to the other tasks was the result of the corrective 424 saccade being delayed. Further, the greater lead of the hand when online cursor feedback was 425 provided resulted from an earlier response of the hand. Finally, correlations between correction 426 latencies differed between tasks, with lower correlations in the classic double-step task and 427 placeholder task than in the overlap, line cue, and arrow cue tasks.

\section{Discussion}

429 This study aimed at determining the effects of visual target and cue characteristics on temporal 430 eye-hand coordination when hand movements had to rapidly correct for a change in movement 431 goal location, while the eyes were free to move. To date, few studies have investigated online 432 corrections of goal-directed reach movements while also measuring eye movements. In addition, 433 the task demands in these studies were limited to simple target step paradigms. We found that 434 across stimulus and task conditions, participants initiated a corrective saccade in addition to 435 correcting the hand trajectory. We report the following key findings: First, the correction latency in 436 eye and hand depended on visual and cognitive processing demands of the task. For example, 437 task versions that contained a cue indicating the change in movement goal triggered longer438 latency corrections as compared to task versions in which participants simply followed the target 
439 when it stepped to a new location. Second, eye and hand corrections were not initiated 440 simultaneously: the hand started correcting before the eye. The hand-eye correction delay was 441 greatest when the target change was signaled by a symbolic cue. Third, correction latencies of 442 the eye and hand were weakly correlated, within individuals and tasks, in target step tasks, and 443 moderately correlated in tasks where the target change was indicated by an additional target or 444 cue, requiring an intentional correction. Fourth, online visual feedback of the hand cursor, 445 compared to endpoint feedback only, sped up the initiation of online corrections of the hand 446 trajectory but did not affect the latency of corrective saccades. Together, our findings show that 447 the timing and coordination of rapid movement corrections of the eye and hand depend on the 448 visual and cognitive demands of the change in movement goal. These findings provide important 449 insights into the role of stimulus and task demands during rapid movement corrections, and will 450 be discussed in detail below.

Our first key finding is that the latencies of both corrective saccades and reach corrections depend on the processing demands of the task. Previous studies already showed that visual target characteristics can affect reach correction latencies in response to a target change (Kozak et al. 2019; Veerman et al. 2008). Here, we show that corrective saccade latencies are also affected by the visual as well as cognitive demands of the task. Specifically, consistent with our hypothesis, corrective saccade latencies increased, relative to those in the classic double-step task, in a placeholder task where all three possible targets were shown and the target swapped position with one of the placeholders, and further increased in an overlap task where the new 459 target was shown in addition to the initial target. Corrective saccade latencies also increased 460 relative to the classic double-step task when the target change was indicated by a spatial (line) cue, and further increased when the target was indicated by a symbolic (arrow) cue. The latencies of corrections of the reach trajectory showed a similar pattern, but without a further increase in latency in the arrow cue task. Note that in all of our tasks, reach correction latencies were slower than commonly reported. This is most likely a result of the extra mass and inertia of the robotic manipulandum, but could also have been caused by relatively low contrast of stimuli on the screen (Veerman et al. 2008). Nevertheless, the task-dependency of latencies emphasizes the importance of visual characteristics of the target and the requirements of the task for the timing of movement corrections.

Our second key finding is that corrections in eye and hand are not initiated simultaneously,

470 and-as the absolute latency-the relative latency of corrections depends on the visual and 471 cognitive processing demands of the task. Whereas many previous studies reported that the eye 472 typically leads the hand when initiating a movement (Bowman et al. 2009; Land and Hayhoe 2001; 
473 Neggers and Bekkering 2000; Prablanc et al. 1979), here we show that the opposite can be true 474 when performing a correction, as Abekawa and colleagues (2014) have previously shown for 475 corrective movements in response to a target displacement (see also Gritsenko et al. 2009). 476 Although the relative latencies between corrections of the hand and the eye were around zero in 477 most of the tasks in the current study, it is important to note that even in cases where corrections 478 of the eye and hand were detected at around the same time from their velocities, the longer 479 neuromuscular delay and greater inertia of the arm and hand imply that the neural signal to 480 execute a correction was sent earlier to the arm muscles than to the eye muscles. The finding 481 that the reach correction is initiated before the corrective saccade indicates that the initial 482 correction of the reach must be based on an approximate computation of the visual movement 483 goal location (Franklin et al. 2016), since high-resolution retinal information as well extraretinal 484 information of the new target position does not become available until the eye lands on the new 485 target. By contrast, the later part of the correction could be more refined, as shown for trajectory 486 corrections in response to cursor displacements (Cross et al. 2019). Further, the delay of the corrective saccade was greater in the arrow cue task compared to the other tasks, as a result of a further increase in corrective saccade but not reach correction latencies. In this particular task, the eye appeared to linger, likely reflecting the time it took to process the shape information of the visual cue, requiring the involvement of the ventral visual stream (Goodale and David Milner 1992). Moreover, in the arrow cue task, the cue was presented centrally, akin to tasks involving endogenous visual spatial attention. Such tasks typically involve slower and more sustained shifts of attention. By contrast, the line cue task involved an exogenous cue, which is known to trigger faster, transient shifts of visual spatial attention (Busse et al. 2008; Carrasco 2011). The time course of events in our tasks reflects the differences between endogenous and exogenous visual spatial attention. However, it is somewhat surprising that the extra processing time in the symbolic cue task affected the eye, but did not cause a further increase in reach correction latencies.

As another aspect of temporal eye-hand coordination, we showed that, within individuals and tasks, correction latencies of the eye and hand are weakly to moderately correlated. Our third key finding is that these correlations were higher for tasks that involved a slower, intentional or 501 voluntary correction (overlap and cue tasks) than in tasks that involved a faster, more automatic 502 correction (classic double-step and placeholder tasks). This is similar to the findings reported by 503 Sailer and colleagues (2000) for direct goal-directed actions. Generally, correlations in movement 504 latencies imply a link in the mechanisms driving these movements (de Brouwer et al. 2021). 505 Higher correlations in more demanding tasks might indicate an overlap in the latency distribution 506 of eye and hand movements for voluntary but not for reactive saccades, or might indicate the 
presence of a 'bottleneck' in information processing that affects both the oculomotor and the limbmotor system (though this would not explain the greater hand-eye delay in the arrow cue task). Even though correction latencies were overall correlated, the initiation of eye and hand corrections is likely driven by separate movement commands subserved by different neural circuitry (de Brouwer et al. 2021).

Our fourth key finding is that online visual feedback on the hand (cursor) location, as compared to endpoint feedback only, sped up the initiation of hand movement corrections (Reichenbach et al. 2009), resulting in a greater lead of the hand. Corrective saccade latencies did not differ between blocks with online and endpoint cursor feedback This was in contrast to our hypothesis that online cursor feedback would (also) reduce the latency of corrective saccades, given the fact that directing the eyes to the reach target improves monitoring of the reach trajectory in peripheral vision (de Brouwer et al. 2018), providing an important reason to move the eyes to the new target location as soon as possible. Presumably, cursor feedback increases certainty about the location of the hand (Acerbi et al. 2017; Izawa and Shadmehr 2008), and therefore accelerates the correction, but this facilitation does not transfer to the eye. studies on online corrections of reaching movements have emphasized the role of the posterior parietal cortex (Desmurget et al. 1999; Pisella et al. 2000), as well as the possibility of a subcortical pathway guiding both the eye and the limb when performing corrections (Day and Brown 2001; Reynolds and Day 2012) (see also Cross et al. 2019; Pruszynski et al. 2010). The exact contributions of these areas are still unknown (for reviews see Archambault et al. 2015; Gaveau et al. 2014). Reynolds and Day proposed that a subcortical circuit allows for very fast responses, while a cortical pathway drives slightly slower but more flexible responses. Although our results cannot provide conclusive evidence about the pathways involved in online corrections, the short latencies in the classic double-step task and longer latencies in the other tasks are consistent with the idea of a subcortical and cortical circuit. Further, the involvement of the ventral visual stream can explain the additional increase in latencies in the arrow cue task.

In summary, we designed a set of double-step tasks and showed that the correction of the reach trajectory in response to a target change is accompanied by a corrective saccade. Both the

536 absolute and relative latencies of the corrective saccade and reach correction depended on the 537 visual characteristics of the target change. Corrections were initiated later when the task required 538 more visual and cognitive processing, and the hand typically started correcting before the eye, 539 especially when the change was initiated by a symbolic cue. Our results highlight that the hand 540 and eye are coordinated in a flexible manner that is suited for the task at hand. Our findings also 
541 provide a framework for discussing latency differences obtained across tasks and studies and

542 emphasize the importance of taking stimulus and task conditions into account when assessing

543 eye-hand coordination. This is especially important when aiming to generalize laboratory results

544 to the more visually and cognitively demanding conditions of real-world tasks.

545 Acknowledgements

546 We would like to thank Hannah Brown for help with data collection. This work was funded by an 547 NSERC Discovery Grant and Accelerator Supplement (RGPIN 418493) awarded to Miriam 548 Spering.

550 Abekawa N, Inui T, Gomi H. Eye-hand coordination in on-line visuomotor adjustments.

$551 \quad$ Neuroreport 25: 441-445, 2014.

552 Acerbi L, Vijayakumar S, Wolpert DM. Target Uncertainty Mediates Sensorimotor Error 553 Correction. PLoS One 12: e0170466, 2017.

554 Archambault PS, Ferrari-Toniolo S, Caminiti R, Battaglia-Mayer A. Visually-guided

555 correction of hand reaching movements: The neurophysiological bases in the cerebral cortex.

556 Vision Res 110: 244-256, 2015.

557 Becker W, Jürgens R. An analysis of the saccadic system by means of double step stimuli.

558 Vision Res 19: 967-983, 1979.

559 Bowman MC, Johansson RS, Flanagan JR. Eye-hand coordination in a sequential target 560 contact task. Exp Brain Res 195: 273-283, 2009.

561 Brenner E, Smeets JB. Fast Responses of the Human Hand to Changes in Target Position. J 562 Mot Behav 29: 297-310, 1997.

563 Brenner E, Smeets JBJ. Fast corrections of movements with a computer mouse. Spat Vis 16: 564 365-376, 2003.

565 de Brouwer AJ, Flanagan JR, Spering M. Functional Use of Eye Movements for an Acting 566 System. Trends Cogn Sci 25: 252-263, 2021.

567 de Brouwer AJ, Gallivan JP, Flanagan JR. Visuomotor feedback gains are modulated by 568 gaze position. J Neurophysiol 120: 2522-2531, 2018.

569 Busse L, Katzner S, Treue S. Temporal dynamics of neuronal modulation during exogenous 570 and endogenous shifts of visual attention in macaque area MT. Proc Natl Acad Sci U S A 105:

571 16380-16385, 2008. 
Carpenter RHS. Movements of the Eyes. 1977.

573 Carrasco M. Visual attention: the past 25 years. Vision Res 51: 1484-1525, 2011.

574 Cousineau D. Confidence intervals in within-subject designs: A simpler solution to Loftus and

575 Masson's method. Tutorials in Quantitative Methods for Psychology 1: 42-45, 2005.

576 Cross KP, Cluff T, Takei T, Scott SH. Visual Feedback Processing of the Limb Involves Two 577 Distinct Phases. The Journal of Neuroscience 39: 6751-6765, 2019.

578 Day BL, Brown P. Evidence for subcortical involvement in the visual control of human reaching. 579 Brain 124: 1832-1840, 2001.

580 Day BL, Lyon IN. Voluntary modification of automatic arm movements evoked by motion of a visual target. Experimental Brain Research 130: 159-168, 2000.

Desmurget M, Epstein CM, Turner RS, Prablanc C, Alexander GE, Grafton ST. Role of the posterior parietal cortex in updating reaching movements to a visual target. Nat Neurosci 2:

584 563-567, 1999.

Diedrichsen J, Nambisan R, Kennerley SW, Ivry RB. Independent on-line control of the two hands during bimanual reaching. Eur J Neurosci 19: 1643-1652, 2004.

587 Franklin DW, Reichenbach A, Franklin S, Diedrichsen J. Temporal Evolution of Spatial 588 Computations for Visuomotor Control. J Neurosci 36: 2329-2341, 2016.

589 Franklin DW, Wolpert DM. Specificity of reflex adaptation for task-relevant variability. $J$ 590 Neurosci 28: 14165-14175, 2008.

591 Gaveau V, Pisella L, Priot A-E, Fukui T, Rossetti Y, Pélisson D, Prablanc C. Automatic online control of motor adjustments in reaching and grasping. Neuropsychologia 55: 25-40, 5932014.

Georgopoulos AP, Kalaska JF, Massey JT. Spatial trajectories and reaction times of aimed movements: effects of practice, uncertainty, and change in target location. $J$ Neurophysiol 46: 725-743, 1981.

Goodale MA, David Milner A. Separate visual pathways for perception and action. Trends in Neurosciences 15: 20-25, 1992.

Goodale MA, Pelisson D, Prablanc C. Large adjustments in visually guided reaching do not depend on vision of the hand or perception of target displacement. Nature 320: 748-750, 1986.

604 Gritsenko V, Yakovenko S, Kalaska JF. Integration of predictive feedforward and sensory 605 feedback signals for online control of visually guided movement. J Neurophysiol 102: 914-930, 6062009.

607 Hallett PE, Lightstone AD. Saccadic eye movements towards stimuli triggered by prior 

of flexible and general motor learning. Exp Eye Res 114: 6-15, 2013.

611 Izawa J, Shadmehr R. On-line processing of uncertain information in visuomotor control. J

612 Neurosci 28: 11360-11368, 2008.

613 Joiner WM, Fitzgibbon EJ, Wurtz RH. Amplitudes and directions of individual saccades can

614 be adjusted by corollary discharge. J Vis 10: 22.1-12, 2010.

615 Kozak RA, Kreyenmeier P, Gu C, Johnston K, Corneil BD. Stimulus-Locked Responses on 616 Human Upper Limb Muscles and Corrective Reaches Are Preferentially Evoked by Low Spatial 617 Frequencies. eNeuro 6, 2019.

618 Land MF, Hayhoe M. In what ways do eye movements contribute to everyday activities? Vision 619 Res 41: 3559-3565, 2001.

620 Magescas F, Prablanc C. Automatic drive of limb motor plasticity. J Cogn Neurosci 18: 75-83, 6212006.

622 McLaughlin SC. Parametric adjustment in saccadic eye movements. Perception \& 623 Psychophysics 2: 359-362, 1967.

Megaw ED. Possible modification to a rapid on-going programmed manual response. Brain Res 625 71: 425-441, 1974.

626 Mrotek LA, Soechting JF. Target interception: hand-eye coordination and strategies. J Neurosci 27: 7297-7309, 2007.

628 Neggers SF, Bekkering H. Ocular gaze is anchored to the target of an ongoing pointing 629 movement. J Neurophysiol 83: 639-651, 2000.

630 Neggers SFW, Bekkering H. Coordinated control of eye and hand movements in dynamic 631 reaching. Hum Mov Sci 21: 349-376, 2002.

632 Oostwoud Wijdenes L, Brenner E, Smeets JBJ. Analysis of methods to determine the latency of online movement adjustments. Behav Res Methods 46: 131-139, 2014. ataxia. Nat Neurosci 3: 729-736, 2000.

Prablanc C, Echallier JF, Komilis E, Jeannerod M. Optimal response of eye and hand motor systems in pointing at a visual target. I. Spatio-temporal characteristics of eye and hand 35: 113-124, 1979.

641 Prablanc C, Martin O. Automatic control during hand reaching at undetected two-dimensional 642 target displacements. J Neurophysiol 67: 455-469, 1992.

643 Pruszynski JA, Andrew Pruszynski J, King GL, Boisse L, Scott SH, Randall Flanagan J, 644 Munoz DP. Stimulus-locked responses on human arm muscles reveal a rapid neural pathway 645 linking visual input to arm motor output. European Journal of Neuroscience 32: 1049-1057, 6462010. 
Oostwoud Wijdenes L, Brenner E, Smeets JBJ. Comparing Online Adjustments to Distance and Direction in Fast Pointing Movements. Journal of Motor Behavior 45: 395-404, 2013.

Reichenbach A, Franklin DW, Zatka-Haas P, Diedrichsen J. A dedicated binding mechanism for the visual control of movement. Curr Biol 24: 780-785, 2014.

Reichenbach A, Thielscher A, Peer A, Bülthoff HH, Bresciani J-P. Seeing the hand while reaching speeds up on-line responses to a sudden change in target position. J Physiol 587:

653 4605-4616, 2009.

654 Reynolds RF, Day BL. Direct visuomotor mapping for fast visually-evoked arm movements. 655 Neuropsychologia 50: 3169-3173, 2012.

656 Ross J, Morrone MC, Goldberg ME, Burr DC. Changes in visual perception at the time of 657 saccades. Trends Neurosci 24: 113-121, 2001.

658 Sailer U, Eggert T, Ditterich J, Straube A. Spatial and temporal aspects of eye-hand coordination across different tasks. Exp Brain Res 134: 163-173, 2000.

Sarlegna F, Blouin J, Bresciani J-P, Bourdin C, Vercher J-L, Gauthier GM. Target and hand position information in the online control of goal-directed arm movements. Exp Brain Res 151: 524-535, 2003.

Saslow MG. Effects of Components of Displacement-Step Stimuli Upon Latency for Saccadic Eye Movement. Journal of the Optical Society of America 57: 1024, 1967.

665 Saunders JA, Knill DC. Humans use continuous visual feedback from the hand to control fast 666 reaching movements. Exp Brain Res 152: 341-352, 2003.

667 Singh T, Perry CM, Herter TM. A geometric method for computing ocular kinematics and 668 classifying gaze events using monocular remote eye tracking in a robotic environment. $J$ 669 Neuroeng Rehabil 13: 10, 2016.

670 Smeets JB, Oostwoud Wijdenes L, Brenner E. Movement Adjustments Have Short Latencies 671 Because There is No Need to Detect Anything. Motor Control 20: 137-148, 2016.

672 Soechting JF, Lacquaniti F. Modification of trajectory of a pointing movement in response to a 673 change in target location. J Neurophysiol 49: 548-564, 1983.

674 Tian J, Ethier V, Shadmehr R, Fujita M, Zee DS. Some perspectives on saccade adaptation. 675 Ann N Y Acad Sci 1164: 166-172, 2009.

676 Tian J, Ying HS, Zee DS. Revisiting corrective saccades: Role of visual feedback. Vision 677 Research 89: 54-64, 2013.

678 Veerman MM, Brenner E, Smeets JBJ. The latency for correcting a movement depends on the 679 visual attribute that defines the target. Exp Brain Res 187: 219-228, 2008. 